\title{
The natural history of ventricular septal defects
}

\author{
S W Turner, S Hunter, J P Wyllie
}

\begin{abstract}
Aims-To correlate the size and position of isolated ventricular septal defects with closure rate in a cohort of children with mean follow up of more than six years.

Design-A birth cohort was identified using the northern region cardiac database. The following were noted from case notes: defect size, position, means of closure, and age at closure.

Results-68 children were identified. 49 defects were small, 14 were moderate, and 5 were large. 13 cases required surgical closure, including 12 perimembranous defects. 35 defects closed spontaneously. Nine of the small muscular defects remained open and five of the small perimembranous defects remained open. The spontaneous closure rate for muscular defects was significantly greater than for perimembranous defects. Mean age of follow up for patients who still have defects is 76 months.

Conclusions-The position of a ventricular septal defect is extremely relevant to its natural history. Perimembranous defects accounted for most of the moderate and large defects that required surgical intervention. After more than six years almost a third of all perimembranous and just over two thirds of all muscular defects closed spontaneously.

(Arch Dis Child 1999;81:413-416)
\end{abstract}

Keywords: ventricular septal defects; cohort study; colour flow imaging

Ventricular septal defects (VSDs) are the second most common congenital heart defect after bicuspid aortic valves, ${ }^{1}$ yet their true incidence is surprisingly unknown. Similarly, their natural history, including incidence of spontaneous closure, is uncertain.

After cross sectional echocardiography became an established means of diagnosing VSDs, ${ }^{2}$ the incidence apparently increased from $1.35-4.0 / 1000$ live births ${ }^{3-5}$ to 3.6-6.5/ 1000 live births. ${ }^{5-7}$ However, cross sectional echocardiography has some limitations in detecting smaller VSDs and the gold standard for VSD diagnosis has been shown to be cross sectional echocardiography with colour flow imaging. ${ }^{8-11}$ Studies that have not used both cross sectional echocardiography and colour flow mapping might not detect all VSDs and will therefore not present an accurate VSD incidence.

Two recent studies using both cross sectional echocardiography and colour flow mapping have screened healthy, asymptomatic newborn infants and found a greatly increased VSD incidence of $2-4.2 \% .^{12}{ }^{13}$ Therefore, it appears that there is a very high incidence of asymptomatic small muscular VSDs, which close spontaneously.

The natural history of individual defects has already been studied in patient cohorts using cross sectional echocardiography and colour flow mapping. ${ }^{14}{ }^{15}$ The longest time that a cohort has been studied so far is 48 months. ${ }^{15}$ Large VSDs and those associated with Down's syndrome or heart failure are less likely to close spontaneously. ${ }^{11}{ }^{14}$ Smaller defects and muscular defects tend to close spontaneously more frequently than others. ${ }^{74}$ The probability of spontaneous closure can be given for a population but it has not been possible to predict closure in individual cases.

We aimed to try to answer questions regarding the incidence and natural history of clinically detectable VSDs. Our report sets out the methodology of a longitudinal, population based cohort study. We report our findings after a mean follow up of 76 months.

\section{Methods}

Our study was carried out in the UK northern region, which has well defined boundaries and a stable population of 3.1 million. All paediatric centres except one small unit refer to the only regional paediatric cardiology centre (Freeman Hospital). At the end of 1990, all the referring paediatricians in the region were informed in writing of our study, and all neonatologists were informed at a regional meeting. The consultants were asked to refer any infant or child known or suspected to have a VSD immediately to the regional cardiothoracic centre.

The Freeman Hospital has a database that contains all details of congenital cardiac defects in live born children. Details of all neonatal and infant deaths where a diagnosis of VSD was made were obtained from the Regional Maternity Survey Office. Using these databases, we examined case notes for all children born in 1991 diagnosed as having an isolated VSD, although patients with the following coexisting conditions were included: dextrocardia, spontaneously closing patent foramen ovale, and spontaneously closing arterial duct.

One of us (ST) reviewed the case notes and noted the reported VSD size and position from the echocardiography report at diagnosis. Hewlett Packard Sonos 1000 and ATL Ultramark 9 echocardiography machines were used with dedicated paediatric transducers and colour flow mapping. The defects were categorised as muscular (apical, trabecular, or outlet), perimembranous (subaortic or subpulmonary), or doubly committed subarterial, according to the classification of Gatzoulis et al. ${ }^{16}$ 
Closure was said to have occurred in one of three situations: (1) at surgical intervention; (2) when neither echocardiography nor colour flow mapping could identify a defect; (3) when VSD was no longer audible, having been detected previously on Doppler or colour flow mapping. Children who had not had cross sectional echocardiography after 72 months of age were recalled for repeat echocardiography.

One of us $(\mathrm{SH})$ examined original video recordings blinded to the outcome and clinical information. An assessment of size and position was made in an attempt to counter inter-individual variation on the original echocardiography reports. VSDs were deemed to be large if the defects were as large as or greater than the aortic orifice, and small if only seen in some parts of the cardiac cycle or not seen at all but identified on colour flow mapping. All other defects were classified as moderate.

\section{Results}

Sixty eight cases were identified in live born children. All patients had cross sectional echocardiography confirmation of their VSD at diagnosis. There were a further three isolated VSDs in stillborn children (two with Edwards syndrome).

Thirty five of the children were boys. The children were first seen at a median age of 2.5 months with a range of 2 days to 42 months. Five children were born before 36 weeks' gestation.

One child died (after successful closure of a medium sized muscular VSD). There were three cases associated with Down's syndrome (all three required surgical closure of large perimembranous VSDs). Three VSDs were associated with arterial ducts or patent oval foramina, which closed spontaneously. There was one VSD associated with isolated dextrocardia. Three of the patients had multiple VSDs.

One child with a small patent muscular defect was lost to follow up at 16 months. Three children with small patent muscular defects not seen in clinic after 72 months of age

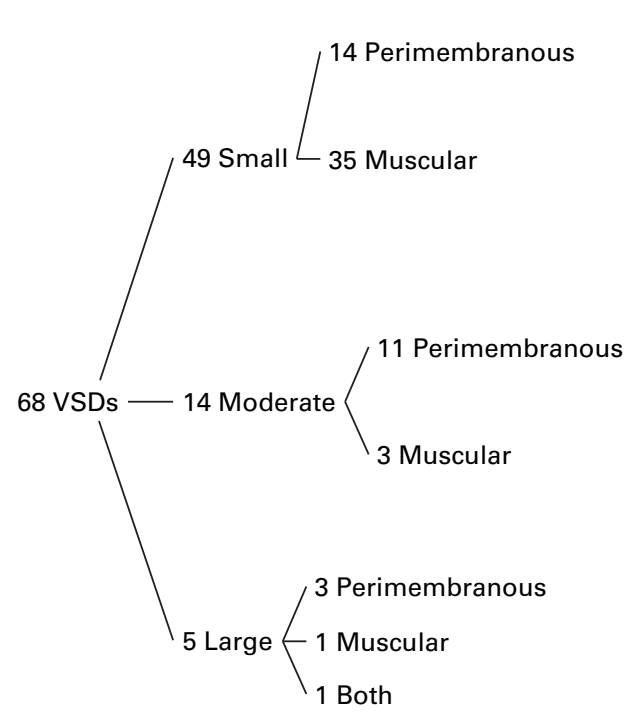

8 Closed spontaneously

5 Remain patent

1 Closed surgically

25 Closed spontaneously 10 Remain patent (1 lost)

4 Remain patent 7 Closed surgically

2 Closed spontaneously 1 Closed surgically

3 Closed surgically

1 Remains patent

1 Closed surgically

Figure 1 Flow chart showing the natural history of the 68 ventricular septal defects (VSDs) studied.

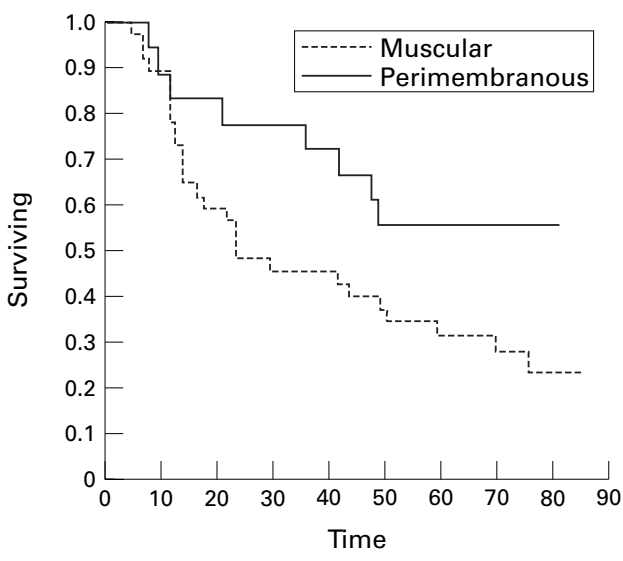

Tests between groups

$\begin{array}{lccc}\text { Test } & \chi^{2} & \text { df } & \text { Prob }>\chi^{2} \\ \text { Log-Rank } & 4.1746 & 1 & 0.0410 \\ \text { Wilcoxon } & 3.3477 & 1 & 0.0673\end{array}$

Figure 2 Kaplan-Meier survival curve comparing spontaneous closure rates of perimembranous and muscular ventricular septal defects. Because figures beyond a mean of 76 months are not complete, the curves are not accurate beyond that point.

declined to attend for echocardiography. They were last seen at 66,67 , and 69 months of age, respectively, and are included in our study.

Figure 1 summarises the natural history of the VSDs.

Forty nine VSDs were small, of which 35 were muscular; 14 were moderate of which three were muscular; there were five patients with large VSDs (including one muscular VSD with multiple small VSDs and one patient with both a large perimembranous defect and a large muscular defect). There were no doubly committed VSDs, which is not surprising because these defects are rare other than in the Far East.

Thirteen VSDs required surgical closure including one patient with two large defects. None required pulmonary artery banding. The VSDs in all but one surgical case were medium or large and in all except one they were perimembranous. One apparently small perimembranous VSD was closed surgically in the presence of heart failure and failure to thrive.

Two cases of moderate perimembranous VSD were closed surgically at 20 and 41 months of age when the concomitant aortic valve prolapse was also repaired. One patient with a spontaneously closing small perimembranous VSD developed right ventricular outflow obstruction that required operative intervention at 49 months of age.

Of the 54 patients managed non-surgically (one having been lost to follow up), 35 defects had spontaneously closed: 27 were muscular and eight were perimembranous. Of the 19 VSDs that did not require surgical closure and remain open, 10 are muscular and nine are perimembranous. Log rank analysis comparing the spontaneous closure rates of muscular and perimembranous defects shows that the difference is significant $(p=0.04$ ) (fig 2 ).

Of the 60 VSDs diagnosed on cross sectional echocardiography at our unit, we reviewed the video recording in 39 instances. In four cases 
there was a discrepancy in size between the original and our echocardiography interpretation. In each case, the retrospective sizing was larger than on contemporaneous reporting. We used the size as described on our original echocardiography report because contemporaneous imaging is probably more accurate than retrospective viewing of images. There was no discrepancy in the position of the VSDs described initially and on retrospective viewing.

\section{Discussion}

This is a small cohort with a long follow up and tight assessment and diagnostic criteria. The results were in part predictable and in part unexpected. We expected the increased spontaneous closure rate of muscular defects. Shirali and colleagues ${ }^{14}$ studied 156 cases for a mean of 28 months and also found a significantly higher spontaneous closure rate for muscular defects.

In general, perimembranous defects are larger than muscular defects, require surgical intervention more frequently, and even when small have little better than a $50 \%$ chance of spontaneous closure by six years. Again we expected this and although our numbers were small this trend of larger defects persisting was in keeping with other less robust studies. ${ }^{317}$ If the closure rate for this group remains unchanged for the next five or six years then one could predict that if a perimembranous defect is not closed by 10 years it is unlikely to close. This is important because there is a continuing risk of developing aortic regurgitation in perimembranous defects. ${ }^{18}$ There are also implications for endocarditis prophylaxis. Gersony et al suggested that the incidence of bacterial endocarditis complicating VSDs is $14.5 / 10000$ patient years. ${ }^{19}$

In 1991 there were 38592 live births in the northern region. There were 68 confirmed cases of isolated VSD in live born infants, and 265 cases of congenital heart defects were registered on the cardiac database. This gives an incidence of isolated VSD of 1.76/1000 live births and a frequency of all congenital heart defects of 6.6/1000. Our VSD frequency was clearly much lower than other post echocardiography studies, which was unexpected, so it is worthwhile considering the causes.

Our study was performed in a stable population with one tertiary cardiology centre and was linked to the longest running fetal abnormality database in the UK. We have examined registers of neonatal deaths and infant deaths for children born in the region in 1991. Consultant general paediatricians and neonatologists participated readily in our study. We have to assume that most VSDs in the region have been detected. All infants in the region have several medical examinations in infancy. It is known that junior doctors can detect up to $50 \%$ of all congenital cardiac defects at the neonatal examination alone, ${ }^{20}$ but that also a large number of life threatening congenital heart defects are missed. ${ }^{21}$ This low incidence might reflect missed diagnoses.

Two recent studies found a very high frequency for isolated VSDs when term neonates were routinely investigated using echocardiography. ${ }^{12}{ }^{13}$ These very high numbers were of VSDs that were neither clinically detectable nor relevant. Most of the defects were small and muscular. In one of the studies, ${ }^{12} 78 \%$ had closed by 6 months of age (A Sands, personal communication, 1998) and $76 \%$ had closed by the 1 st birthday in the second study. ${ }^{13}$ In comparison, our population was identified by using clinical criteria and our study is a population study of more clinical relevance.

There was one case in our cohort of a perimembranous VSD that appeared to be small on imaging yet needed surgical closure as a result of heart failure. Clearly, there are features other than size estimated by cross sectional echocardiography that produce the haemodynamic effect of VSDs. We should remember that we are studying a three dimensional hole in only one or two views so it might be helpful prospectively to measure the defect in more than one orthogonal plane. Three dimensional echocardiography might provide a solution but at present it is not widely available or user friendly. ${ }^{22}$

In the 13 patients with defects so small that they were only seen on colour flow mapping ("tiny" VSDs), none needed surgery and nine of 12 closed spontaneously within six years, with one case lost to follow up. These are clearly defects with a good prognosis. Both of the perimembranous defects in this group closed whereas at least two of the 10 muscular defects remain open. A much larger study is needed before we can confirm that this is a trend.

Thirty nine per cent of perimembranous VSDs required surgical closure and only $29 \%$ closed spontaneously by 6 years of age. This compares with figures of $3 \%$ and $69 \%$, respectively, for muscular VSDs. Perimembranous VSDs are in many ways defects with a poor prognosis.

Our study of VSDs continues and will include the next two years of isolated VSDs. It is hoped that larger numbers and a longer follow up will provide the answers to some of the questions raised by our present study.

We are grateful to Dr M Askenasy and Professor J Matthews for their statistical advice; in addition, we thank the parents and children who attended early echocardiography examination for the purpose of this study.

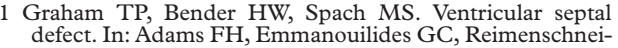
der TA, eds. Moss' heart disease in infants, children and adolescents, 4th ed. Baltimore: Williams and Wilkins, adolescents, 4th

2 Sutherland GR, Godman MJ, Smallhorn JF, Guiterras P, Anderson RH, Hunter S. Ventricular septal defects. Two dimensional echocardiographic and morphological correlations. Br Heart F 1982;47:316-28.

3 Hoffman JIE, Rudolph AM. The natural history of ventricular septal defects in infancy. Am $\mathcal{F}$ Cardiol 1965;16: 634-53.

4 Dickinson DF, Arnold R, Wilkinson JL. Ventricular septal defects in children born in Liverpool 1960 to 1969 . Evalu-
ation of natural course and surgical implications in an unselected population. Br Heart $\mathcal{f}$ 1981;46:47-54.

5 Meburg A, Otterstad JE, Froland G, Sorland S, NitterHauge S. Increasing incidence of ventricular septal defects caused by improved detection rate. Acta Paediatr 1994;83: 653-7.

6 Mehta VA, Chidambaram B. Ventricular septal defect in the first year of life. Am $\mathcal{F}$ Cardiol 1992;70:364-6. 
7 Moe DG, Guntheroth WG. Spontaneous closure of uncomplicated ventricular septal defect. Am 7 Cardiol 1987;60: plicated

8 Helmcke F, de Souza A, Nanda NC, et al. Two-dimensional and color doppler assessment of ventricular septal defect of congenital origin. Am f Cardiol 1989;63:1112-16.

9 Ludomirsky A, Huhta JC, Vick W, III, Murphy DJ, Danford DA, Morrow WR. Color doppler detection of multiple ventricular septal defects. Circulation 1986;74:1317-22.

10 Hornberger LK, Sahn DJ, Krabill KA, et al. Elucidation of the natural history of ventricular septal defects by serial doppler color flo

11 Pieroni DR, Nishimura RA, Bierman FZ, et al. Second natural history of congenital heart defects. Ventricular septal defect: echocardiography. Circulation 1993;87(suppl I):80-8.

12 Sands A, Lynch C, Casey F, Craig B, Dornan J, Mulholland C. Ventricular septal defects; the relationship of social class and area of residence to occurrence rate [abstract]. Fetal Diagn Ther 1998;13(suppl I):148.

Diagn Ther 1998;13(suppl I): 148 .
13 Hiraishi S, Agata Y, Nowatari M, et al. Incidence and natural course of trabecular ventricular septal defect; two dimensional echocardiography and color doppler flow imaging study.

14 Shirali GS, O'Brian Smith E, Geva T. Quantitation of echocardiographic predictors of outcome in infants with isolated ventricular septal defect. Am Heart $\mathcal{F}$ 1995;130 $1228-35$
15 van den Heuvel F, Timmers T, Hess J. Morphological, haemodynamic, and clinical variables as predictors for management of isolated ventricular septal defects. Br Heart f 1995;73:49-52.

16 Gatzoulis MA, Li J, Ho SY. The echocardiographic anatomy of ventricular septal defects. Cardiology in the Young 1997;7: $471-84$

17 Campbell M. Natural history of ventricular septal defects. Br Heart f 1971;33:246-57.

18 Karpawich PP, Duff DF, Mullins CE, Cooley DA, McNamara DG. Ventricular septal defect with associated aortic valve insufficiency-progression of insufficiency and aortic valve insufficiency-progression of insufficiency and
operative results in young children. $\mathcal{f}$ Thorac Cardiovasc operative results in
Surg $1981 ; 82: 182-9$

19 Gersony WM, Hayes CJ, Driscoll DJ, et al. Bacterial endocarditis in patients with aortic stenosis, pulmonary stenosis or ventricular septal defects. Circulation 1993;87(suppl I):1121-6.

20 Ainsworth S, Wyllie JP, Wren C. Prevalence and clinical significance of murmurs in neonates. Arch Dis Child Fetal Neonatal Ed 1999;80:F43-6.

21 Abd-Harb M, Hey E, Wren C. Death in infancy from unrecognised congenital heart disease. Arch Dis Child 1994;71:3-7.

22 Silberbach M. Three-dimensional echocardiographic reconstruction: from "ice-pick" view to virtual reality [editorial]. Mayo Clin Proc 1993;68:311-12. 